\title{
Evaluation of the one-trial passive avoidance task as a tool for studying ECS-produced amnesia'
}

\section{J. P. J. PINEL, ${ }^{2}$ McGill University, Montreal, Canada}

Rats were placed on a "live" grid in the white compartment of a two-compartment box and were allowed to escape to the safe, black compartment before being removed from the box. Each rat was returned to the white compartment $5 \mathrm{sec}$, $1 \mathrm{~min}$, or $24 \mathrm{~h}$ later. Ss, rather than avoiding, "froze" in the white compartiment. The prevalence of this freezing increased with the duration of the test-retest interval. These results are discussed with regard to the use of the one-trial passive avoidance task in studying ECS-produced amnesia.

Much of the knowledge that we have about the physiological nature of memory comes from the study of the effect of electroconvulsive shock (ECS) on the memory of one-trial passive avoidance training in the rat. This task has been used extensively for two main reasons. First, because it is a passive avoidance task, any possible punishing effects of ECS will not summate with any possible amnesic effects. Second, because learning occurs in just one trial, it is possible to specify the exact time of learning, and thus the exact duration of the learning-ECS interval.

However, it seems now that the very nature of this task may be responsible for complicating the study of ECS-produced amnesia. In the one-trial passive avoidance situation, Ss are given a single noxious foot shock for making a particular response, and subsequently refrain from repeating that response. The name one-trial passive avoidance implies that in one trial $S$ learns which response leads to punishment, and then learns to avoid repeating that response. However, it is equally likely that $\mathbf{S}$ just learns a simple conditioned emotional response (CER) or "freezing" response to the apparatus in general, and thus suppresses all responses indiscriminately.

In the one-trial passive avoidance situation, it is difficult to discriminate between these two alternatives because both lead to a decrease in the performance of the negatively reinforced response and an increase in response latency. However, it is important for the interpretation of the many studies which have employed the one-trial passive avoidance task to know which of these two vastly different kinds of memory a rat has after a single avoidance trial. Thus, in the present study these two possible effects of a single punishing electric shock were studied in opposition in a one-trial active avoidance situation. METHOD

The 46 male, experimentally naive, $200 \mathrm{~g}$, black-hooded rats that served as Ss were tested in a simple two-compartment box which consisted of two $8 \times 12$ in. compartments joined by a 3 $x 3$ in. door which remained open throughout the experiment.

On the first two days of testing, each $S$ was placed in the middle of the white compartment facing away from the door. After each rat had shuttled to the black compartment, his latency was recorded and he was removed from the apparatus.

On the third day of testing, the grid floor of the white compartment was "live" with a $2 \mathrm{~mA}$ ac current. Each rat was placed on the live grid, allowed to escape to the safe, black compartment, and removed from the apparatus $10 \mathrm{sec}$ later. Ten rats were returned to the white compartment $5 \mathrm{sec}$ later, 10 were retumed $1 \mathrm{~min}$ later, and 11 were returned $24 \mathrm{~h}$ later. The 24-h Ss spent the intervening time in their home cages. The Ss in the other groups spent this time in their carrying cages. Three control groups consisting of five Ss each were treated in exactly the same was as the three experimental groups except that they were not shocked. No shocks were administered during retesting. During retesting the latency of the shuttling responses was recorded for each $S$. The $S$ s that did not shuttle within $5 \mathrm{~min}$ were removed from the apparatus and given the maximum possible latency score of $300 \mathrm{sec}$.

\section{RESULTS}

The results are presented graphically in Fig. 1. Because there were no significant differences $(p>.05)$ between the mean latencies of the three no-shock control groups at any stage of the experiment, their scores were combined for purposes of analysis.

It can be seen from Fig. 1 that there were no significant differences between the mean latencies of any of the groups on the day before avoidance training $(p>.05)$.

All but one of the Ss that received a footshock exhibited increased rather than decreased latencies after one active avoidance trial. In addition, the postshock latencies of each of the three groups that received an avoidance trial were significantly greater $(p<.05)$ than the combined latencies of the no-shock groups.

The magnitude of this increase in latency became greater as the duration of the test-retest interval increased. The results of a Duncan's Range Test indicated that the postshock latencies of the 24-h group were greater than those of the other two experimental groups $(\mathrm{p}<.05)$. Even though this difference is a large one, as can be seen from Fig. 1, it would have been much larger had the maximum possible score been more than 300 sec. In the 5-sec group only one $S$ failed to avoid in the 5-min retest period and was given a score of $300 \mathrm{sec}$, whereas seven Ss in the 24 h group failed to avoid within $5 \mathrm{~min}$. These results provide still another demonstration that the response to a single, noxious stimulus does incubate or increase in strength over time.
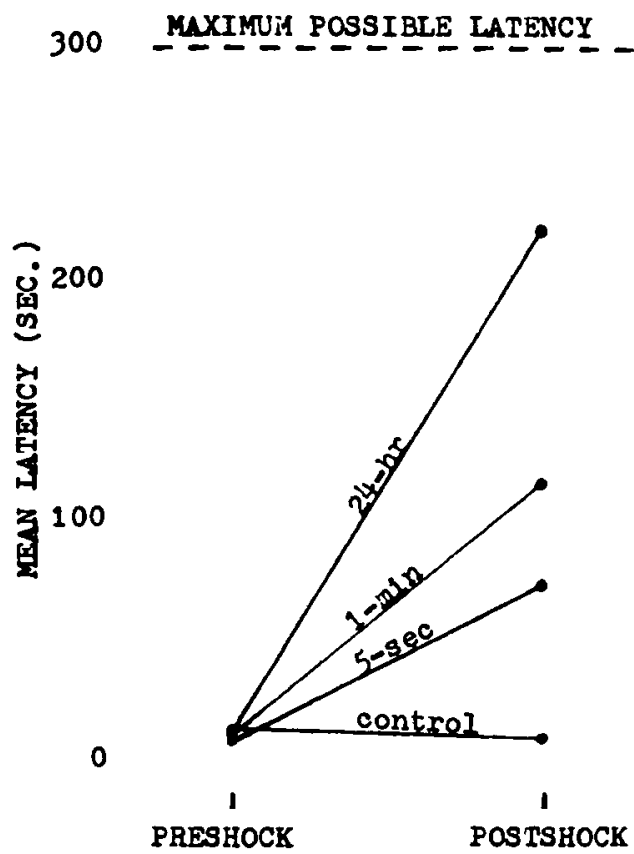

Fig. 1. Latencies before and after one active avoidance trial as a function of test-retest interval. 


\section{DISCUSSION}

The results of the present experiment demonstrate two characteristics of one-trial avoidance that have undoubtedly added to the complexities involved in the study of ECS-produced amnesia.

First. it appears that at least in some cases the name one-trial passive avoidance is a misnomer. In the present experiment. Ss learned a CER rather than an avoidance response after a single punishment. The problem with the one-trial passive avoidance is that it is difficult to be entirely sure which of these two vastly different types of learning is predominant (Spevack \& Suboski, 1967) since both types of learning result in increased latencies to respond and in decreased rates of responding. This is an important consideration since Chorover \& Schiller (1966) suggest that the effects of ECS on these two types of learning may be entirely different. They contend that ECS can "erase" the avoidance memory if administered within seconds of training but that ECS may produce a similar appearing but entirely different effect on the CER which can occur even when ECS is administered hours after training. A recent paper by Kopp, Bohdanecky, \& Jarvik (1960) has cast some doubt on this proposal of Chorover and Schiller. They found that ECS could disrupt a relatively well-discriminated avoidance response when administered hours after avoidance training.

Second. the present study shows as have several other previous studies (Hudson, 1950: Pinel \& Cooper, 1966a, b; Suboski. Spevack. Litner. \& Beaumaster, 1968) that the one-trial avoidance response incubates or increases in strength over time. This greatly complicates the interpretation of any effects ECS might produce on a one-trial avoidance response (McGaugh. 1966: Pinel \& Cooper. 1966b). Hudson's (1950) detailed descriptions of the behavior of the rat after it receives a single punishing shock are quite enlightening in this regard. He reports that the postshock beliavior of rats consists almost entirely of approaching and exploring the area in which they had just been shocked. Occasionally there was no marked evidence of even excitement, suggesting the possibility that some thing had misfired. only to find intense avoidance activity to have developed by the next day even though no further shocks had been administered. The difficulties raised by this and other denonstrations of the incubation phenomenon for the interpretation of the aninesic effects of ECS on one-trial passive avoidance learning are clear. Sometimes the memory of a single. punishing shock is demonstrated by an exploratory approach response. Sometimes retention of the shock is demonstrated by avoidance.

Some investigators have suggested that the graded retroactive effects of ECS on one-trial avoidance training may be related to the incubation of the one-trial avoidance response. Both Pinel \& Cooper (1966b) and Suboski et al (1968) have provided evidence to suggest that ECS interrupts the incubation of the one-trial avoidance response rather than producing any amnesia per se.

Recently investigators have been becoming aware of some of the problems involved in using the one-trial passive avoidance task and have turned to the use of a one-trial discriminated avoidance (Pfingst \& King, 1967: Suboski et al, 1968). This task has the advantage of forcing the animal to learn a specific avoidance response rather than just a general CER. Perhaps an even more fruitful approach would be to escape the complexities of avoidance behavior entirely and use a one-trial appetitive task (Tenen, 1965; Herz, 1967) to gauge the amnesic effects of ECS. This type of learning more closely approximates those types of learning that are known to be disrupted by ECS in human Ss.

\section{REFERENCES}

CHOROVER, S. L., \& SCHILLER, P. H. Reexamination of prolonged retrograde amnesia in one-trial learning. Journal of Comparative \& Physiological Psychology, 1966, 61, 34-41.

HERZ, M. J. Interference with one-trial appetitive and aversive learning by ether and ECS. Proceedings of the 75th Annual Convention of the American Psychological Association, 1967, 2, 83-84.

HUDSON, B. B. One trial learning in the domestic rat. Genetic Psychology Monographs, 1950, 41, 99-145.

IRWIN, S., \& BENUAZIZI, A. Pentylenetetrazol enhances memory function. Science, 1966, 152, 100-102.

IRWIN, S., KALSNER, S., \& CURTIS, A. Direct demonstration of consolidation of one-trial learning. Federation Proceedings, 1964, 23, 102.

KOPP, R., BOHDANECKY, Z., \& JARVIK, M. E. Long temporal gradient of retrograde amnesia for a well-discriminated stimulus. Science, 1966, 153, 1547-1549.

McGAUGH, J. L. Time-dependent processes in memory storage. Science, $1966,153,1351-1358$.

PINEL, J. P. J., \& COOPER, R. M. Incubation and its implications for the interpretation of the ECS gradient effect. Psychonomic Science, 1966a, 6, 123-124.

PINEL, J. P. J., \& COOPER, R. M. The relationship between incubation and ECS gradient effects. Psychonomic Science, 1966b, 6, 125-126.

PFINGST, B. E., \& KING, R. A. A one-trial response-choice technique for the biological study of memory. Psychonomic Science, 1967, 8, 497-498.

SPEVACK, A. A., \& SUBOSKI, M. D. A confounding of conditioned suppression in passive avoidance: ECS effects. Psychonomic Science 1967, 9, 23-24.

SUBOSKI, M. D., SPEVACK, A. A., LITNER, J., \& BEAUMASTER, E. Effects of ECS following one-trial discriminated avoidance conditioning. Neuropsychologia, in press.

TENEN, S. S. Retrograde amnesia from electroconvulsive shock in a one-trial appetitive learning task. Science, 1965, 148, 1248-1250. NOTES

1. Supported by Canadian National Research Council Grant No. APA-66 awarded to Dr. P. M. Milner.

2. Holder of a Canadian National Research Council Scholarship. 